\title{
Mamíferos no voladores en un campus universitario de la Orinoquia colombiana
}

\author{
Andrés Alfonso \\ Grupo de investigación ECOTONOS, Programa de Biología, Facultad de Ciencias Básicas e Ingeniería, Universidad de los Llanos. \\ km 12 vía Puerto López, vereda Barcelona, Villavicencio, Meta, Colombia \\ caaz.alfonso@gmail.com \\ Francisco Sánchez \\ Grupo de investigación ECOTONOS, Programa de Biología, Facultad de Ciencias Básicas e Ingeniería, Universidad de los Llanos. \\ km 12 vía Puerto López, vereda Barcelona, Villavicencio, Meta, Colombia
}

\section{Resumen}

En la Orinoquia colombiana la transformación del paisaje es evidente y se ha sugerido que los campus de instituciones educativas ayudan a conservar la biodiversidad. Por ello estudiamos la composición y la riqueza de los mamíferos no voladores de un campus de la Orinoquia colombiana; además evaluamos la efectividad de tres diferentes cebos. El campus incluye construcciones, bosques secundarios y áreas rurales. Usamos tres tipos de trampas (trampas Sherman, trampas Tomahawk, trampas artesanales tipo Tomahawk) y cámaras trampa; complementamos el inventario con recorridos y entrevistas. Registramos 18 especies y los órdenes con mayor riqueza fueron Rodentia y Primates, mientras que Didelphis marsupialis fue la especie más registrada. El tocino fue más efectivo que la yuca o el maíz para capturar mamíferos. A pesar de las perturbaciones presentes en el campus, éste aun preserva características que, al menos por el momento, le permiten ser hábitat para varias especies de mamíferos silvestres.

Palabras clave: Inventario, Mammalia, piedemonte llanero, riqueza de especies.

\begin{abstract}
In the Colombian Orinoquia landscape transformations are evident and it has been suggested that campuses of educational institutions can help to conserve biodiversity. Consequently, we studied the composition and species richness of non-flying mammals in a campus in the Colombian Orinoquia; we also evaluated the effectiveness of three different baits. The campus is a mosaic of built areas, secondary forest remnants, and rural areas. We used three types of traps (Sherman, Tomahawk, and Tomahawk-like artisanal traps) and complemented the inventory with walks searching for mammal sightings and interviews. We recorded 18 species and the richest orders were Rodentia and Primates. Didelphis marsupialis was the most common species, and bacon was the most effective bait in comparison with corn seeds and cassava roots. Despite the present perturbation in the campus, it still preserves characteristics that, at least for the moment, make it a habitat for several species of wild mammals.
\end{abstract}

Keywords: Inventory, Mammalia, Andean piedmont, species richness.

\section{Introducción}

A comienzos del siglo XX, en la zona de piedemonte llanero colombiano alrededor de Villavicencio, departamento del Meta, había una cobertura abundante de bosque húmedo tropical que se mezclaba con sabanas naturales (Bates, 1948). Esta zona ha sufrido profundos cambios debido a la expansión urbana, agropecuaria y minera, y cada vez hay menos áreas de ecosistemas naturales para las especies que habitan en el que es hoy el mayor centro de actividad económica de la Orinoquia colombiana (Rincón et al. 2010, Santana, 2010, World Wildlife Fundation et al. 2016). Las intervenciones humanas en el piedemonte amenazan la biodiversidad ya que pueden generar límites para las especies silvestres y afectar la estructura de las comunidades biológicas al exponerlas a posibles efectos de borde (Murcia, 1995, Cadenasso et al. 2003). Villavicencio y el piedemonte llanero son solo un ejemplo de una situación que se repite a nivel mundial (Ellis et al. 2010) y por ello algunos han indicado la necesidad de aprovechar los espacios dominados por la actividad humana para desarrollar estrategias para la conservación de la biodiversidad (Rosenzweig, 2003, Hobbs et al. 2011).

Mammalogy Notes | Notas Mastozoológicas

Sociedad Colombiana de Mastozoología

Vol. 5 Núm. 2| 2019 
Se ha argumentado que áreas rurales manejadas apropiadamente pueden ayudar a la conservación de la biodiversidad en áreas neotropicales (Harvey et al. 2008, Otero \& Onaindia, 2009). Algunos autores incluso sugieren que es posible aprovechar espacios urbanos para la conservación de la biodiversidad (Dearborn \& Kark, 2010, Kawarik, 2011). En este respecto, estudios en varias regiones de Colombia indican que, a pesar de las profundas modificaciones en los paisajes que incluyen campus universitarios, estos espacios académicos pueden incluirse en estrategias de conservación. Esto debido a que pueden tener coberturas que se asemejan a ecosistemas naturales (Ramírez-Chaves et al. 2010, Téllez-Farfán et al. 2013, Sánchez et al. 2015) y además la gente allí tiene, en general, una actitud positiva hacia la fauna (Bohner \& Dickel, 2011). Con esto en mente, se propuso estudiar a los mamíferos terrestres presentes en un campus universitario de la Orinoquia colombiana, específicamente el campus Barcelona de la Universidad de los Llanos que se ubica en el piedemonte llanero. En donde hoy es el campus, los bosques, sabanas y humedales naturales prácticamente desaparecieron al establecerse una finca ganadera a inicios del siglo XX, y desde 1974 la perturbación se intensificó con la construcción de edificios para el funcionamiento de la Universidad (Arango, 2010). Sin embargo, el campus se encuentra en un contexto rural-urbano donde han ocurrido también procesos de revegetalización con árboles nativos y exóticos (Suárez L. y Quiñonez L. com. pers.). Estas acciones abren posibilidades para mejorar la calidad del hábitat para la vida silvestre, pero para manejar apropiadamente el espacio es necesario entender mejor los aspectos básicos del ensamblaje de mamíferos terrestres que allí se encuentra.

La composición y riqueza de especies son aspectos fundamentales de la estructura de un ensamblaje, y para estudiar la diversidad de mamíferos no voladores neotropicales es necesario el uso de múltiples técnicas, e incluso diferentes cebos, debido a la amplia variedad de hábitos alimentarios y de movimiento que poseen (Voss \& Emmons, 1996). En este sentido, la mantequilla de maní y el tocino se han reportado como cebos efectivos para la captura de roedores y marsupiales, mientras que los carnívoros prefieren cebos como pescado y carne, mostrando que el tipo de cebo afecta el éxito de captura de mamíferos neotropicales (Woodman et al. 1996, Astúa et al. 2006, Hice \& Velazco, 2013). Lo anterior sugiere que el uso de cebos distintos implica diferencias en el éxito de captura de diferentes mamíferos, y hasta donde nuestra revisión de la literatura indica, para la Orinoquia colombiana no se han realizado evaluaciones del uso de diferentes cebos para atraer mamíferos. Con base en todo lo anterior, el presente estudio empleamos múltiples técnicas de estudio para contestar las siguientes preguntas: 1) ¿Cuál es la composición y riqueza de las especies de mamíferos no voladores presentes en el campus de la universidad?, y 2) ¿cuáles son los cebos más efectivos para capturar mamíferos en el campus?

\section{Metodología}

Área de estudio

El campus de la Universidad de los Llanos se ubica en el km 12 vía Puerto López, vereda Barcelona, municipio de Villavicencio, Meta, Colombia (4,07497, -73,58509, 400 m altitud; Figura 1). Tiene una temperatura anual promedio del aire de $25,9^{\circ} \mathrm{C}$, y una precipitación anual promedio de $3638 \mathrm{~mm}$, con un régimen de lluvias monomodal, con altas precipitaciones entre abril y octubre, y con una humedad relativa del 88,5\% en promedio anual (Minorta \& Rangel-Ch, 2012). El campus incluye pastizales, zonas cultivadas, estanques para piscicultura y áreas construidas. Hay parches de árboles producto de procesos de revegetalización en los que se usaron una mezcla de flora nativa y exótica. También hay áreas asociadas a cursos de agua con remanentes de bosques regenerados naturalmente, pero donde también se han plantado árboles. Las técnicas de registro se aplicaron en las áreas del campus con la mayor cantidad de cobertura arbórea (Figura 1), dado que son las más parecidas a la cobertura vegetal que solía existir antes de la intervención humana (Bates, 1948).

\section{Métodos}

Capturamos mamíferos empleando trampas mecánicas tipo Sherman, Tomahawk, trampas artesanales tipo Tomahawk (Voss \& Emmons, 1999) y cámaras trampa (O'Connell et al. 2010). Realizamos tres sesiones de muestreo, cada una de 15 días, en la estación lluviosa a partir de junio hasta mediados de octubre de 2016. En cada sesión usamos 36 trampas Sherman (16,5 × $5 \times 6,5 \mathrm{~cm})$, tres trampas Tomahawk $(25 \times 30 \times 82 \mathrm{~cm})$, tres trampas artesanales tipo Tomahawk y seis cámaras trampa Bushnell Trophy Cam®. Las cámaras trampa las ubicamos entre 60 y $150 \mathrm{~cm}$ del suelo, y las configuramos para grabar 20 segundos de video, con sensor normal y con un tiempo de espera de 2 segundos (Díaz \& Payán, 2012). Las trampas Sherman y Tomahawk las distribuimos en doce estaciones de captura. Cada una de las doce estaciones contaba con tres trampas

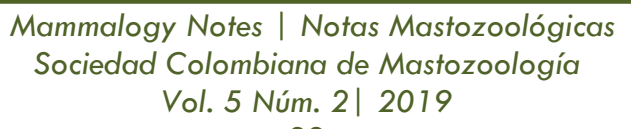


Sherman, que estuvieron a no más de tres metros de distancia entre sí, y estaciones consecutivas estaban a 20 m de distancia. Seis de las estaciones con trampas Sherman también tenían una trampa Tomahawk o artesanal, que estaban a 20 m de distancia de las Sherman. Las cámaras las situamos en un área diferente del campus a la de las otras trampas (Figura 1), y allí había a una distancia de al menos 35 m entre estaciones consecutivas, y cada estación contaba con una sola cámara.

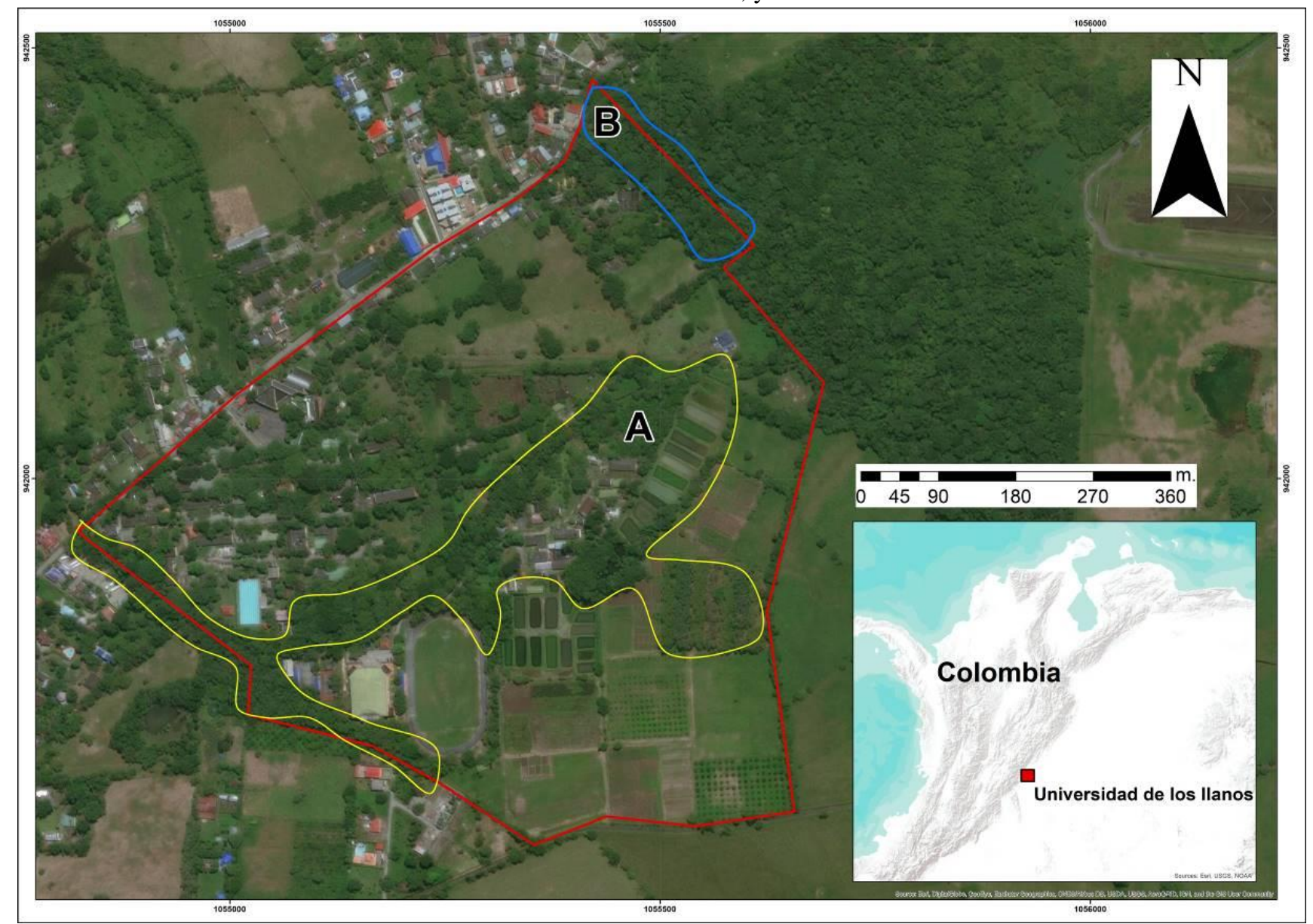

Figura 1. Campus de la Universidad de los Llanos ubicado en Villavicencio, departamento del Meta, en el piedemonte llanero de la Orinoquia colombiana. A. Área de muestreo con los tres tipos de trampa (Sherman, Tomahawk y Cámaras trampa; área delineada en amarillo). B. Área de muestreo solo con cámaras trampa (área delineada en azul).

Utilizamos como cebos semillas de maíz, trozos de yuca y trozos de tocino. Usamos 4 a 5 cubos de $\sim 1 \mathrm{~cm}^{3}$ de yuca, de 4 a 5 granos de maíz, o 3 cubos pequeños de tocino para cada trampa Sherman. Para las trampas Tomahawk, preparamos paquetes con 15 semillas de maíz, yuca o tocino envueltos en gaza. A su vez, dejamos granos de maíz, trozos de yuca y tocino en el área de visión de las cámaras trampa. Estos cebos se han utilizado con anterioridad para atraer mamíferos neotropicales (Woodman et al. 1996, Astúa et al. 2006, Hice \& Velazco, 2013). En cada cámara trampa, Tomahawk o artesanal colocamos un tipo de cebo cada día, y cada cinco días los cambiamos por un nuevo cebo hasta completar 15 días. En cada estación donde ubicamos las trampas Sherman ofrecimos los tres tipos de cebos, cada trampa tenía un cebo diferente, y cada cinco días rotamos las trampas de una estación. Así, cada trampa o cámara tuvo los tres cebos durante tres períodos de cinco días cada uno, hasta completar un total de 45 días de muestreo. Revisamos todos los días las trampas Sherman, artesanales y Tomahawk, y recebamos con el alimento correspondiente para ese día. Las cámaras trampa funcionaron de manera continua durante 15 días, y cada cinco días cambiamos los cebos. En las cámaras, consideramos que los animales eran atraídos por el cebo cuando los observamos en el video olfatearlo o comerlo. Los individuos que no realizaron estos comportamientos sólo los usamos para complementar el inventario y estimar la riqueza de especies. En el tiempo entre muestreos, las cámaras trampa 
funcionaron continuamente sin cebos para complementar el inventario. Así, en total funcionaron durante 100 noches; 45 con cebo y 55 sin cebo.

Con el fin de complementar el inventario, entrevistamos al personal de seguridad, de servicios generales, docentes y estudiantes de la Universidad sobre la presencia de mamíferos en el campus. Además, registramos los avistamientos hechos durante los recorridos para revisar las trampas. Estos recorridos, los realizó A. Alfonso en horas de la mañana, ca. 06:00 hr, y en horas de la tarde, ca. 18:00 hr. También, los mamíferos que encontramos muertos fueron recolectados. A su vez, revisamos la colección de mamíferos del Museo de Historia Natural de la Universidad de los Llanos por especímenes recolectados en el campus. Los ejemplares de mamíferos del campus de las especies que no estaban disponibles en el Museo, los recolectamos y depositamos allí mismo (Anexo 1). Los animales no recolectados los liberamos en el sitio de captura. Identificamos a los animales con literatura especializada (Emmons \& Feer, 1999, Gardner, 2008, Patton et al. 2015). Usamos información de la UICN (2016) y la resolución N 1912 del Ministerio de Ambiente y Desarrollo Sostenible de 2017 para categorizar los mamíferos registrados según su estado de conservación.

Calculamos el éxito de captura como el número de capturas dividido por el esfuerzo de muestreo, que se obtuvo como la multiplicación del número de trampas por el número de días que funcionaron. Además, utilizamos el programa EstimateS 9.1.0 para obtener curvas de acumulación de especies y estimar la riqueza total de especies utilizando los estimadores no paramétricos de Chao 1, Jacknife 1 y Bootstrap (Colwell 2014). La efectividad de los cebos fue comparada mediante una prueba de Kruskall-Wallis, seguida por una prueba de Mann-Whitney con corrección de Bonferroni para hacer comparaciones múltiples (Zar 2010). Todas las pruebas estadísticas las evaluamos con un nivel de significancia de $\alpha=0,05$.

\title{
Resultados
}

Con las trampas Sherman, Tomahawk, artesanales y cámaras trampa obtuvimos un total de 191 registros de mamíferos silvestres, de los cuales diez fueron capturados con cebos en las trampas Sherman y Tomahawk (Anexo 2). El método más efectivo fue el de cámaras trampa, con 184 registros, de los cuales 85 fueron con cebos. Por lo tanto, usando los tres tipos de trampas logramos registrar nueve especies, y en total usando todos métodos registramos 18 especies de mamíferos nativos, pertenecientes a siete órdenes (Anexo 2, Figura 1). En los recorridos observamos tres especies de Primates, dos roedores, un marsupial (Didelphis marsupialis), un oso hormiguero y un venado (Anexo 2). Por otro lado, con las entrevistas documentamos la presencia de erizos (Coendou sp.), chuchas (Didelphis marsupialis), armadillos (Dasypus sp.) y zorros (Cerdocyon thous). Además, cinco especies fueron registradas por medio de fotografías o videos facilitados por estudiantes y docentes (Anexo 3). Todas las especies registradas no están consideradas en ninguna categoría de amenaza, con excepción de tres especies (Anexo 1).

Las curvas de acumulación de especies usando los datos de trampas Sherman, Tomahawk, artesanales y cámaras por 45 días con cebos, y de sólo cámaras por 100 días no lograron nivelarse. Además, las dos curvas de acumulación de especies no indicaron diferencias significativas en el número de especies registradas (Figura 2). La riqueza total estimada con los datos de trampas Sherman, Tomahawk, artesanales y cámaras por 45 días según el estimador Jacknife 1 fue de alrededor de nueve especies $(8,96 \pm 1,37)$, y cerca de 12 según el estimador Chao 1 (intervalo de confianza del 95 \% [IC 95\%]: 7,06 - 17,89). La riqueza estimada sólo con las cámaras por 100 días fue de alrededor de 12 especies según el estimador de Jackknife 1. (11,97 $\pm 1,7$ ) y alrededor de 21 especies según Chao 1 (IC 95\% 9,36 - 33,92). Esto indica que la eficiencia de muestreo fue aproximadamente de alrededor del $58-77 \%$ con los tres tipos de trampas y aproximadamente $43-75 \%$ con solo cámaras trampas.

\author{
Mammalogy Notes | Notas Mastozoológicas \\ Sociedad Colombiana de Mastozoología \\ Vol. 5 Núm. 2| 2019
}




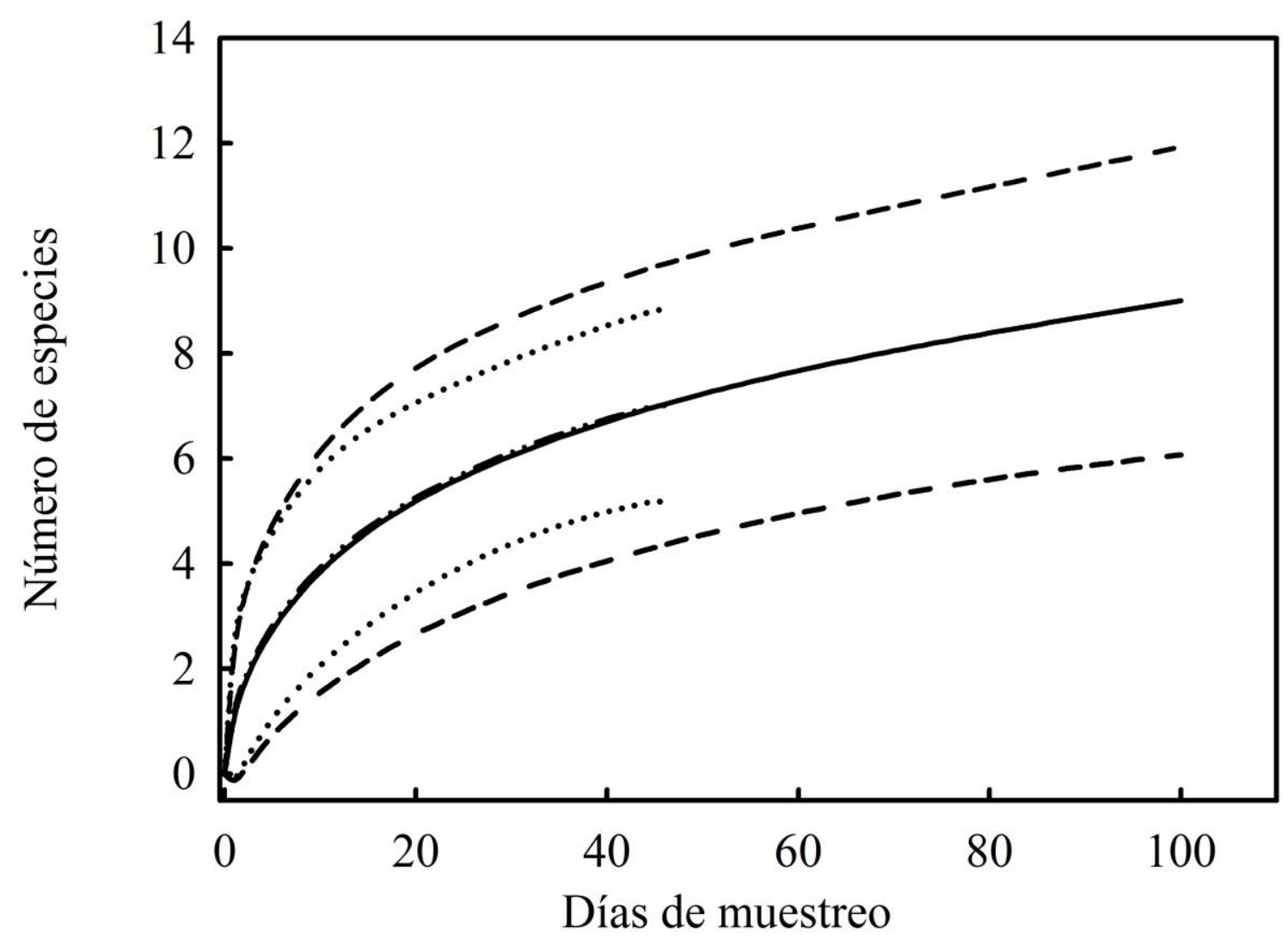

Figura 2. Curvas de acumulación de especies de mamíferos en la Universidad de los Llanos usando trampas Sherman, Tomahawk y cámaras trampa por 45 días con cebos (línea discontinua-punteada; intervalo de confianza $95 \%$ : líneas punteadas), y sólo cámaras trampa por 100 días (línea sólida; intervalo de confianza 95 \%: líneas discontinuas).

Hubo diferencias significativas en el éxito de captura entre los tres diferentes cebos utilizados $\left(\chi^{2}=13,98, \mathrm{gl}=2, \mathrm{p}<0,01\right)$. El tocino tuvo un mayor éxito de captura respecto a los demás cebos (maíz-tocino $U=669, \mathrm{p}<0,01$; tocino-yuca $U=638$, $p$ $<0,01$ ), y atrajo una especie de marsupial (D. marsupialis), posiblemente tres especies de roedores (Cuniculus paca, Rattus rattus y una especie no identificada), un armadillo (Dasypus novemcinctus) y un zorro (Cerdocyon thous). No hubo diferencias entre el éxito de captura del maíz y la yuca $(\mathrm{U}=994, \mathrm{p}=0,86$; Figura $3 \mathrm{~A})$. El 73,4\% de los registros con todas las trampas fueron de D. marsupialis, y de nuevo el tipo de cebo afectó el éxito de captura de este marsupial $\left(\chi^{2}=13,29, \mathrm{gl}=2, \mathrm{p}<0,01\right)$. El tocino fue el más eficaz (maíz-tocino $U=700, p<0,01$; tocino-yuca $U=671, p<0,01$ ) y no hubo diferencias entre el maíz y la yuca $(\mathrm{U}=994, \mathrm{p}=0,84$; Figura $3 \mathrm{~B})$.

Registramos cuatro especies exóticas en el campus: perros (Canis lupus familiaris, $\mathrm{n}=11)$ y gatos (Felis silvestris catus, $\mathrm{n}=$ 6) en recorridos y cámaras trampa en las zonas periféricas del campus, donde era más abundante la cobertura arbórea. Además, dos ratas (R. rattus) y un ratón (Mus musculus) fueron capturados en trampas Sherman. 

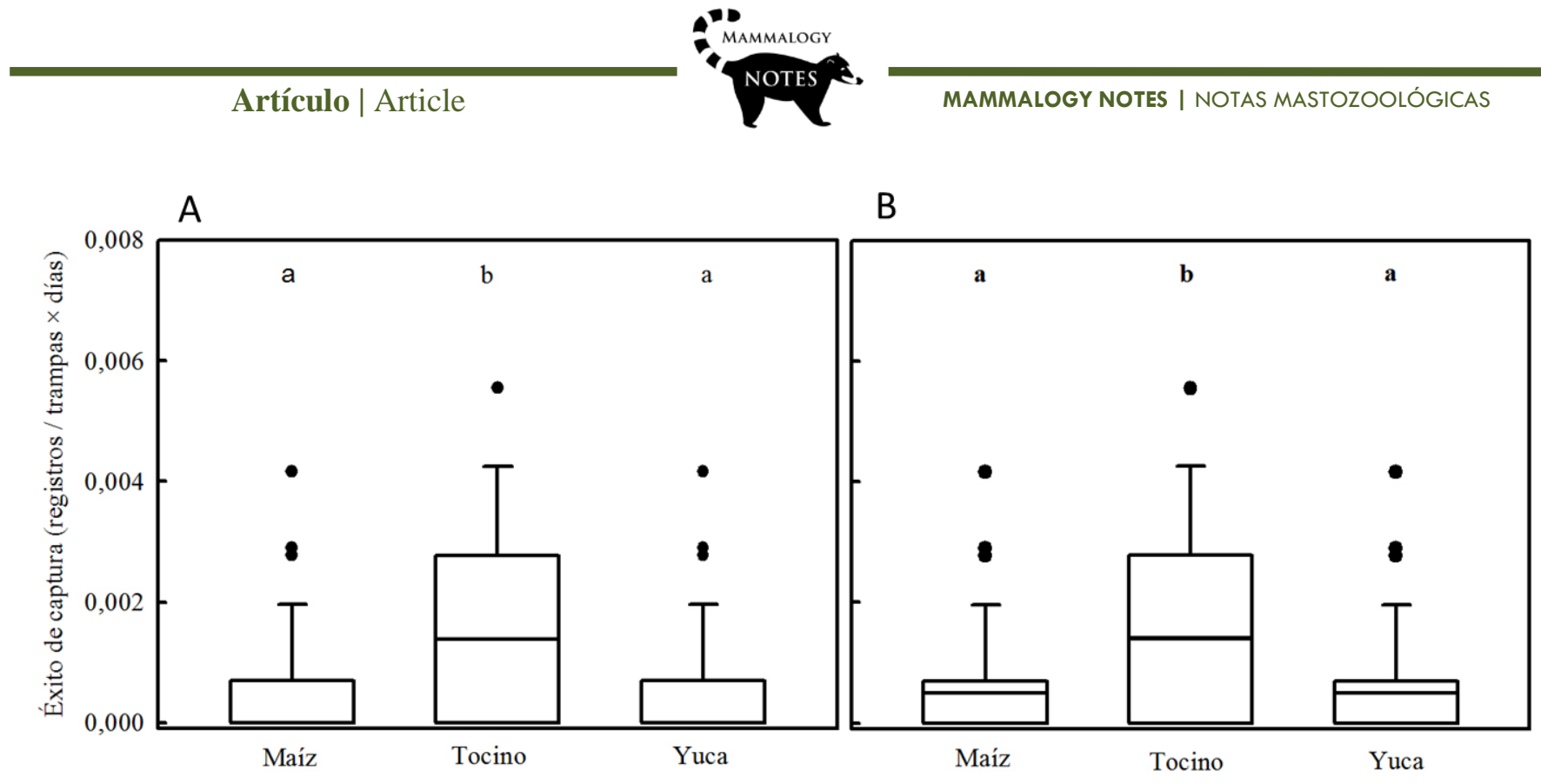

Figura 3. Éxito de captura de mamíferos en el campus. A) Éxito de captura de todos los mamíferos en el campus de la Universidad de los Llanos usando trampas Sherman, Tomahawk, artesanales y cámaras trampa. B) Éxito de captura de Didelphis marsupialis. La línea dentro de la caja es la mediana, sus extremos son los percentiles 25 y 75 , las barras de error representan los percentiles 10 y 90, y los puntos son valores atípicos. Letras diferentes sobre las cajas denotan diferencias significativas (U de Mann-Whitney con corrección de Bonferroni, $\mathrm{p}<0,05$ ).

\section{Discusión}

Como en otras áreas neotropicales, el uso de múltiples técnicas de muestreo permitió registrar mamíferos con variados patrones de actividad y formas de movilización (Voss \& Emmons 1996, Sánchez et al. 2004). Sin embargo, las curvas de acumulación de especies no se nivelaron y los estimadores de riqueza sugieren que son necesarios muestreos adicionales en el campus. También son recomendables muestreos adicionales para obtener especímenes que permitan verificar la identidad taxonómica de especies como la ardilla del campus. De acuerdo con la información en el Sistema de Información sobre Biodiversidad del Instituto de Investigación de Recursos Biológicos Alexander von Humboldt (SIB, 2017), en el campus podrían faltar otras especies reportadas para Villavicencio como: Galictis vittata, Lontra longicaudis, Pteronura brasiliensis, Bradypus variegatus, Cheracebus torquatus, Lagothrix lagotricha. No obstante, la ausencia de bosques maduros, cuerpos de agua de tamaño suficiente, y la sostenida alteración del campus y sus alrededores debido a la actividad humana no parecen permitir la presencia de estas especies animales. Lamentablemente, dicha perturbación podría incrementarse en un futuro por planes para ampliar las vías de acceso al campus y la construcción de nuevos edificios a expensas de áreas arboladas. Por otra parte, la distribución de C. torquatus se ha restringido a la región amazónica (Defler 2010, Solari et al. 2013), y debe examinarse si la determinación del espécimen reportado en el SIB es correcta, para reconocer si en efecto este primate podría estar en Villavicencio.

Debido a los profundos cambios que han ocurrido en las coberturas naturales de los llanos orientales colombianos (RomeroRuiz et al. 2012, Etter et al. 2017), hace más de 40 años la riqueza de mamíferos era probablemente mayor que la de la actualidad (Boshell, 1938, Morales et al. 1979, D'Alessandro et al. 1981, Wells et al. 1981, D'Alessandro et al. 1984). En efecto, la riqueza de mamíferos no voladores en la Orinoquia colombiana se ha registrado que tiende a disminuir conforme aumenta la intervención humana (Guzmán-Lenis \& Camargo-Sanabria, 2004, Torres, 2010, González, 2014, GonzálezJiménez \& Choque-Ladino, 2015, Pardo-Vargas \& Payán-Garrido, 2015). Así, las profundas modificaciones que han ocurrido en el paisaje del que es parte el campus de la Universidad de los Llanos, y las perturbaciones locales que persisten, probablemente son responsables de que la mayoría de las especies de mamíferos registrados en el campus sean conocidas por tolerar ambientes intervenidos por los humanos (Eisenberg, 1989, Emmons \& Feer, 1999, Morales-Jiménez et al. 2004). La

Mammalogy Notes | Notas Mastozoológicas

Sociedad Colombiana de Mastozoología

Vol. 5 Núm. 2| 2019 
Universidad de los Llanos solía ser una finca ganadera con poca vegetación, pero luego del establecimiento del campus se plantaron árboles y se permitió la regeneración del bosque en algunas zonas, y esto ha permitido que actualmente en el campus se encuentran representados todos los órdenes taxonómicos de mamíferos no voladores de la Orinoquia, exceptuando Lagomorpha y Perissodactyla. Además, en el campus también hay especies amenazadas como Plecturocebus ornatus, Aotus brumbacki y Myrmercophaga tridactyla (UICN 2016, Minambiente 2017), lo que resalta la importancia del campus.

Por otra parte, el tocino fue el cebo más efectivo para capturar mamíferos, lo que concuerda con estudios previos (Astúa et al. 2006). Con este cebo registramos marsupiales, roedores y un cánido. A diferencia de lo reportado previamente (Hice \& Velazco, 2013), la yuca y el maíz tuvieron un bajo éxito de captura, lo que probablemente se deba a la baja frecuencia de pequeños roedores en el campus. La chucha común D. marsupialis fue la especie más registrada usando tocino. Esta especie tiene una amplia distribución en Colombia (Solari et al. 2013: Ramírez-Chaves et al. 2016), y puede habitar en zonas perturbadas (Pérez-Hernández et al. 1994). Además, este marsupial también se registró varias veces con todos los cebos, lo que concuerda con trabajos previos que reconocen la dieta omnívora de la especie (Astúa et al. 2006, Hice \& Velazco, 2013). En el campus son frecuentes humanos y animales domésticos, por lo que conocer el cebo más efectivo para capturar y manejar a D. marsupialis puede ser de utilidad, pues esta especie puede ser reservorio de enfermedades como la leishmaniasis y la enfermedad de Chagas (Herrera, 2010, Aponte, 2013).

Nuestros resultados también sugieren que en zonas intervenidas la probabilidad de capturar especies nativas de tamaño pequeño es baja, y durante el estudio no logramos capturas vivas de ninguna especie nativa de menos de $200 \mathrm{~g}$ de los órdenes Rodentia o Didelphimorphia. Esto concuerda con otros estudios en campus universitarios de Colombia donde no es común la presencia de mamíferos pequeños nativos (Ramírez-Chaves et al. 2010, Téllez-Farfán et al. 2013, García \& Agudelo, 2013, Sánchez et al. 2015). En los Andes argentinos las especies de mamíferos pequeños no nativos tienen un mayor éxito en zonas perturbadas, desplazando o reemplazando a las especies nativas locales (Martin-Albarracin et al. 2015). Así, los resultados sugieren que los pequeños mamíferos son sensibles a la presencia humana y sus actividades, como ha sido reportado en otras localidades (Olifiers et al. 2005, Di Bitetti et al. 2013). Además, la presencia de especies exóticas como ratas, ratones, perros y gatos probablemente está afectando la fauna local vía depredación, transmisión de enfermedades o competencia (Dabritz et al. 2006, Calver et al. 2011; Sierra et al. 2011). Dadas las experiencias disponibles en la literatura, sería recomendable controlar el número de gatos y perros en el campus para disminuir su impacto sobre la fauna silvestre (Lepczyk et al. 2004, Calver et al. 2011). Con el mismo propósito, también sería recomendable utilizar trampeos dirigidos a la captura de roedores exóticos, y que no afecten a las especies silvestres (Taylor \& Thomas, 1993).

Los mamíferos presentes en el campus se enfrentan a la pérdida y degradación de hábitat, causadas por la expansión urbana y rural en Villavicencio (Rincón-Castillo \& Jaramillo-Salazar, 2010, World Wildlife Fundation et al. 2016). Se ha propuesto que en espacios altamente intervenidos por los humanos se pueden hacer modificaciones que ayuden a mejorar la calidad del hábitat para la fauna silvestre (Rosenzweig, 2003). La plantación de árboles en el campus parece estar funcionando hasta el momento, y deberían continuarse con estos esfuerzos. En efecto, la presencia de especies de mamíferos con diferentes hábitos tróficos, patrones de actividad y formas de movilización, sugiere que ha ocurrido un proceso de rehabilitación en el campus asociado al incremento en la cobertura arbórea (Choi, 2007). La revegetalización en el campus probablemente incrementó la disponibilidad de refugios y otros recursos los mamíferos silvestres, y varias especies aprovechan, por ejemplo, los frutos de varios árboles para alimentarse (Datos sin publicar). Entre esos árboles están especies exóticas como mango (Mangifera indica), papaya (Carica papaya), naranja (Citrus $\times$ sinensis), mandarina (Citrus $\times$ mandarina) y pomarrosa $($ Syzygium jambos), además de plantas herbáceas como el banano (Musa $\times$ paradisiaca). Estudios futuros deben examinar la forma en que la vegetación del campus puede permitir o no la preservación de las especies aquí registradas en el mediano y largo plazo.

Por último, la fauna silvestre puede verse afectada por factores a nivel del paisaje, es decir procesos que ocurren alrededor del campus (Roselli \& Stiles, 2012, Rocha et al, 2016). Por ello, idealmente se deben integrar esfuerzos con los vecinos del campus para entender cómo el mosaico de coberturas de la zona podría aprovecharse para permitir la permanencia de los mamíferos silvestres. De particular interés en el futuro sería la generación y mantenimiento de corredores biológicos que

Mammalogy Notes | Notas Mastozoológicas

Sociedad Colombiana de Mastozoología

Vol. 5 Núm. 2| 2019 
conecten los parches de vegetación del campus con bosque cercanos para mantener los mamíferos silvestres del campus (Ortiz-Moreno \& Rodríguez-Pires, 2014). En ambientes altamente intervenidos como el campus esto puede lograrse al adicionar árboles y/o estructuras artificiales que puedan, por ejemplo, facilitar el desplazamiento de las especies (SuárezCáceres et al. en prensa).

\section{Agradecimientos}

Unillanos permitió el desarrollo del trabajo en el campus. A H. Ramírez y J. Astwood, hicieron comentarios a versiones iniciales del manuscrito. Dos evaluadores anónimos también hicieron valiosas contribuciones para mejorar el documento. F. Tique, M. Reyes, E. Fino, A. Morales, M.F. PaQui, F. Lozano y G. Moreno facilitaron fotos y/o videos de mamíferos del campus. C. Torres y V. Rodríguez ayudaron durante el trabajo de campo. D. Rao corrigió el resumen en inglés. El Museo de Historia Natural-Unillanos facilitó algunas de las trampas para realizar este trabajo.

\section{Referencias}

Aponte, J., 2013. Una revisión de la biología del Didelphis marsupialis y su relación con el mal de Chagas y la leishmaniasis. Hipótesis, apuntes científicos uniandinos. 1,96-101. Arango, A., 2010. El nacimiento. Pp 37-46 En 35 años: Universidad de los Llanos, el proyecto estratégico más importante de la historia 1975-2010 (Salazar GO, Ladino N, Castillo E, Arango A, Baquero A, Jiménez CA, Sabogal G, y Camacho N. eds.) Universidad de los Llanos, Villavicencio, Colombia.

Astúa, D., Moura, R.T., Grelle C.E., Fonseca M.T., 2006. Influence of baits, trap type and position for small mammal capture in a Brazilian lowland Atlantic Forest. Boletim do Museu de Biologia Mello Leitão. 19,31-44.

Bates, M., 1948. Climate and vegetation in the Villavicencio region of eastern Colombia. Geogafical Review, 38(4),555-574. https://doi.org/10.2307/211443

Bohner, G. \& Dickel, N. 2011. Attitudes and attitude change. Annual review of psychology 62:391-417. https://doi.org/10.1146/annurev.psych.121208.131609

Boshell, J., 1944. Epidemiologia de la fiebre amarilla selvática en Colombia durante los últimos años. Revista de la Facultad de Medicina Universidad Nacional de Colombia. 13(2), 122-144.

Cadenasso, M.L., Pickett, S.T., Weathers, K. C., Jones, C.G., 2003. A framework for a theory of ecological boundaries. BioScience. 53(8),750-758. https://doi.org/10.1641/00063568(2003)053[0750:AFFATO]2.0.CO;2

Calver, M.C., Grayson, J., Lilith, M., Dickman, C.R., 2011. Applying the precautionary principle to the issue of impacts by pet cats on urban wildlife. Biological Conservation. 144(6),1895-1901. https://doi.org/10.1016/j.biocon.2011.04.015

Choi, Y.D., 2007. Restoration Ecology to the Future: A call for new paradigm. Restoration Ecology. 15(2), 351-353.

Colwell, R.K., 2014. EstimateS: statistical estimation of species richness and shared species from samples version 9. 1. 0. Storrs: University of Connecticut. Recuperado de http://viceroy.colorado.edu/estimates.

Dabritz, H.A., Atwill, E.R., Gardner, I.A., Miller, M.A., Conrad, P.A., 2006. Outdoor fecal deposition by free-roaming cats and attitudes of cat owners and non-owners toward stray pets, wildlife, and water pollution. Journal of the American Veterinary Medical Association. 229,74-81. https://doi.org/10.2460/javma.229.1.74

D'Alessandro, A., Rausch, R.L., Morales, G.A., Collet, S., Angel, D., 1981. Echinococcus infections in Colombian animals. American Journal of Tropical Medicine and Hygiene. 30(6),1263-1276. https://doi.org/10.4269/ajtmh. 1981.30.1263

D'Alessandro, A., Barreto, P., Saravia, N., Barreto, M., 1984. Epidemiology of Trypanosoma cruzi in the Oriental plains of Colombia. American Journal of Tropical Medicine and Hygiene. 33(6),1084-1095. https://doi.org/10.4269/ajtmh.1984.33.1084

Dearborn, D.C., Kark, S. 2010. Motivations for conserving urban biodiversity. Conservation Biology. 24(2), 432-440. https://doi.org/10.1111/j.1523-1739.2009.01328.x Defler, T.R., 2010. Historia natural de los primates colombianos. Universidad Nacional de Colombia, Bogotá, Colombia.

Di Bitetti, M.S., Albanesi, S.A., Foguet, M.J., De Angelo, C., Brown, A.D., 2013. The effect of anthropic pressures and elevation on the large and medium-sized terrestrial mammals of the subtropical mountain forests (Yungas) of NW Argentina. Mammalian Biology. 78,21-27. https://doi.org/10.1016/j.mambio.2012.08.006

Díaz, A., Payán, E., 2012. Manual de fototrampeo: una herramienta de investigación para la conservación de la biodiversidad en Colombia. Instituto de Investigaciones de Recursos Biológicos Alexander von Humboldt y Panthera, Colombia 32.

Eisenberg, J.F., 1989. Mammals of the Neotropics: The northern Neotropics (Vol. 1). Chicago: The University of Chicago Press.

Ellis, E.C., Goldewijk, K.K., Siebert, S., Lightman, D., Ramankutty, N., 2010. Anthropogenic transformation of the biomes, 1700 to 2000. Global Ecology and Biogeography. 19,589-606. https://doi.org/10.1111/j.1466-8238.2010.00540.x

Emmons, L.H., Feer, F., 1999. Mamíferos de los bosques húmedos de América tropical: una guía de campo. Editorial FAN, Santa Cruz de la Sierra 298.

Etter, A., Andrade, A., Saavedra, K., Amaya, P., Arevalo, P., Cortes, J., Pacheco, C., Soler, D., 2017. Lista roja de ecosistemas de Colombia. (Vers. 2.0),1-6.

García, A.M., Agudelo, Y.J., 2013. Gestión Ambiental Universitaria: Construyendo un campus sustentable. Universidad Tecnológica de Pereira, Pereira, Colombia 27.

Gardner, A.L., 2008. Mammals of South America, volume 1: marsupials, xenarthrans, shrews, and bats. The University of Chicago Press, Chicago, E.E.U.U. 690.

González, A.M., 2014. Evaluación ecológica rápida del parque metropolitano María Lucía. Revista Científica Guarracuco. 18(29),1-18.

González-Jiménez, G., Choque-Ladino, N., 2015. Condiciones actuales en términos de pérdida de biodiversidad en corredores biológicos de la Granja Agroecológica Uniminuto, Villavicencio, Meta, Colombia. Revista de Investigación Agraria y Ambiental. 6(1),239-252. https://doi.org/10.22490/21456453.1283

Guzmán-Lenis, A., Camargo-Sanabria, Á., 2004. Importancia de los rastros para la caracterización del uso de hábitat de mamíferos medianos y grandes en el bosque Los Mangos (Puerto López, Meta, Colombia). Acta Biológica Colombiana. 9,11-22.

Harvey, C.A., Komar, O., Chazdon, R., Ferguson, B.G., Finegan, B., Griffith, D.M., Martínez-Ramos, M., Morales, H., Nigh, R., Soto-Pinto, L., Van Breugel, M., Wishnie, M., 2008. Integrating agricultural landscapes with biodiversity conservation in the Mesoamerican hotspot. Conservation Biology. 22,8-15. https://doi.org/10.1111/j.15231739.2007.00863.x

Herrera, L., 2010. Una revisión sobre reservorios de Trypanosoma (Schizotrypanum) cruzi (Chagas 1909), agente etiológico de la Enfermedad de Chagas. Boletín de Malariología y Salud Ambiental. 50,3-15.

Hice, C.L., Velazco, P.M., 2013. Relative effectiveness of several bait and trap types for assessing terrestrial small mammal communities in Neotropical rainforest. Occasional papers, Museum of Texas Tech University. 316,1-16.

Hobbs, R.J., Hallett, L.M., Ehrlich, P.R., Mooney, H.A., 2011. Intervention ecology: applying ecological science in the twenty-first century. BioScience. 61,442-450. https://doi.org/10.1525/bio.2011.61.6.6

Kawarik, I., 2011. Novel urban ecosystems, biodiversity, and conservation. Environmental Pollution. 159,1974-1983. https://doi.org/10.1016/j.envpol.2011.02.022 
Lepczyk, C.A., Dauphine, N., Bird, D.M., Conant, S., Cooper, R.J., Duffy, D.C., Hatley, P., Marra P.P., Stone, E., Temple, S.A., 2010. What conservation biologists can do to counter trap-neuter-return: response to Longcore et al. Conservation Biology. 1,627-629. https://doi.org/10.1111/j.1523-1739.2009.01426.x

Martin-Albarracin, V.L., Nuñez, M.A., Amico, G.C., 2015. Replacement of native by non-native animal communities assisted by human introduction and management on Isla Victoria, Nahuel Huapi National Park. PeerJ. 3,1-19. https://doi.org/10.7717/peerj.1328

Martínez, A., Rangel-Ch, J.O., 2012. Mamíferos de la Orinoquia colombiana. Pp. 751-784 en Colombia Diversidad Biótica XIV. La región de la Orinoquia colombiana (Rangel J. O. ed.). Instituto de Ciencias Naturales, Universidad Nacional de Colombia, Bogotá, Colombia.

Ministerio de Ambiente y Desarrollo Sostenible., 2017. Resolución No 1912 [15 septiembre 2017]. en: http://www.minambiente.gov.co/images/normativa/app/resoluciones/75res\%201912\%20de\%202017.pdf. Consultado:14 jun 2018.

Minorta, V., Rangel-Ch, J.O., 2012. El clima de la Orinoquia colombiana. Pp. 153-206 en Colombia Diversidad Biótica XIV. La región de la Orinoquia colombiana (Rangel J. O. (ed.). Instituto de Ciencias Naturales, Universidad Nacional de Colombia, Bogotá, Colombia.

Morales, G.A., Guzman, V.H., Wells, E.A., Angel, D., 1979. Polycystic echinococcosis in Colombia: the larval cestodes in infected rodents. Journal of Wildlife Diseases. 15,421428. https://doi.org/10.7589/0090-3558-15.3.421

Morales-Jiménez, A. L., Sánchez, F., Poveda, K., \& Cadena, A. (2004). Mamíferos terrestres y voladores de Colombia: guía de campo. Bogotá, Colombia: Ramos López Editorial. Murcia, C., 1995. Edge effects in fragmented forests: implications for conservation. Trends in Ecology and Evolution. 10(2)58-62. https://doi.org/10.1016/S0169-5347(00)889776

O'Connell, A.F., Nichols, J.D., Karanth, K.U., 2010. Camera traps in animal ecology: methods and analyses. Springer, Heidelberg. https://doi.org/10.1007/978-4-431-99495-4 Olifiers, N., Gentile, R., Fiszon, J.T., 2005. Relation between small-mammal species composition and anthropic variables in the Brazilian Atlantic Forest. Brazilian Journal of Biology. 65(3),495-501. https://doi.org/10.1590/S1519-69842005000300015

Otero, J., Onaindia, M., 2009. Landscape structure and live fences in Andes Colombian agrosystems: upper basin of the Cane-Iguaque River. Revista de Biología Tropical. 57(4), 1183-1192.

Ortiz-Moreno, M.L., Rodríguez-Pires, J.S., 2014. Aplicación de la legislación ambiental y territorial en municipios capitales: Estudio de caso Villavicencio (Colombia). Orinoquia, $18(2), 130-148$

Pardo-Vargas, L. E. \& Payán-Garrido, E. 2015. Mamíferos de un agropaisaje de palma de aceite en las sabanas inundables de Orocué (Casanare, Colombia). Biota Colombiana. 16,54-66.

Patton, J.L., D’Elía, G., Pardiñas, U.F.J., 2015. Mammals of South America, Rodents. Vol. 2. University of Chicago Press, Chicago, E.E.U.U. 1136.

Pérez-Hernández, R., Soriano, P., Lew, D., 1994. Marsupiales de Venezuela. PER. 599.22/23 (87).

Ramírez-Chaves, H.E., Perez, W., Egas, O.M., Tosse, H.T., Muñoz, A., Lozada, A.T., 2010. Biodiversidad en el campus de la Universidad del Cauca, Popayán, Colombia. Biotecnología en el Sector Agropecuario y Agroindustrial. 8(2),104-117.

Ramírez-Chaves, H.E., Suárez-Castro, A.F., González-Maya, J.F., 2016. Cambios recientes a la lista de los mamíferos de Colombia. Mammalogy notes. 3(1),1-9.

Rincón A., Jaramillo, C.A., 2010. Establecimiento, manejo y utilización de recursos forrajeros en sistemas ganaderos de suelos ácidos. MADR, Fedegan, CORPOICA. Villavicencio. Colombia 251.

Rocha, R., López-Baucells, A., Farneda, F.Z., Groenenberg, M., Dobrowiec, P.E.D., Cabeza, M., Palmeirim, j., Meyer, C.F.J., 2016. Consequences of a large-scale fragmentation experiment for Neotropical bats: disentangling the relative importance of local and landscape-scale effects. Landscape Ecology. 32(1),31-45. https://doi.org/10.1007/s10980-016$0425-3$

Romero-Ruiz, M.H., Flantúa, S.G.A., Tansey, K., Berrio, J.C., 2012. Landscape transformations in savannas of northern South America: Land use/cover changes since 1987 in the Llanos Orientales of Colombia. Applied Geography. 32(2),766-776. https://doi.org/10.1016/j.apgeog.2011.08.010.

Roselli, L., Stiles, F.G., 2012. Local and landscape environmental factors are important for the conservation of endangered wetland birds in a high Andean plateau. Waterbirds. 35,453-469. https://doi.org/10.1675/063.035.0310

Rosenzweig, M. L. 2003. Win-win ecology: how the Earths species can survive in the midst of human enterprise. Oxford University Press, Chicago, E.E.U.U. 211.

Sánchez, F., Martínez-Habibe, M.C., Díaz, S., Medina, N., Riaño, J., PaQui, M.F., 2015. Biodiversidad en un campus universitario en la sabana de Bogotá: inventario de plantas y tetrápodos. Boletín Científico Museo de Historia Natural. 19(2),186-203. http://dx.doi.org/10.17151/bccm.2015.19.2.11

Sánchez, F., Sánchez-Palomino, P., Cadena, A., 2004. Inventario de mamíferos en un bosque de los Andes centrales de Colombia. Caldasia. 6,291-309. http://dx.doi.org/10.15446/caldasia

Santana Castañeda, E., 2010. El reconocimiento de la biodiversidad regional. Orinoquia. 14,1-2.

SIB. 2017. Sistema de Información sobre Biodiversidad, Instituto de Investigación de Recursos Biológicos Alexander von Humboldt. www.sibcolombia.net. Consultado: 15 May 2017.

Sierra, E., Bolio, M., Altamirano, M., Cocom, E., Hiuit, R., Antúnes, J., 2011. Análisis de la presencia de perros "ferales" sobre la salud ambiental en la Reserva Ecológica "Cuxtal", Mérida, Yucatán, México. Bioagrociencias. 4,53-57.

Solari, S., Muñoz-Saba, Y., Rodríguez-Mahecha, J.V., Defler, T.R., Ramírez-Chaves, H.E., Trujillo, F., 2013. Riqueza, endemismo y conservación de los mamíferos de Colombia. Mastozoología Neotropical. 20(2),301-365.

Suárez-Cáceres, G.P., Adinolfi, C. \& Sánchez, F. en prensa. Food selection and use of space by Didelphis pernigra (Didelphidae: Mammalia) in a suburban Andean environment. Acta Biológica Colombiana 26(1).

Taylor, R.H. Thomas, B.W., 1993. Rats eradicated from rugged Breaksea island (170 ha), Fiordland, New Zealand. Biological Conservation. 65(3),191-198. https://doi.org/10.1016/0006-3207(93)90052-3

Téllez-Farfán, L., Posada-Florez, F., Sánchez, F., 2013. Biodiversidad en un rincón del borde norte de Bogotá. Universidad de Ciencias Aplicadas y Ambientales U.D.C.A., Bogotá, Colombia 146.

Torres, D.A., 2010. Caracterizaciones biológicas en la Hacienda Macondo (Mapiripán, Meta). Orinoquia 14(2):18-27.

UICN. The IUCN Red List of Threatened Species. Version 2016-2. Disponible en: www.iucnredlist.org. Consultado: 29 Nov 2016.

Voss, R.S., Emmons, L.H., 1996. Mammalian diversity in Neotropical lowland rainforests: a preliminary assessment. Bulletin of the American Museum of Natural History. 230,1115 .

Wells, E.A., D'Alessandro, A., Morales, G.A., Angel, D., 1981. Mammalian wildlife diseases as hazards to man and livestock in an area of the Llanos Orientales of Colombia. Journal of Wildlife Diseases. 17,153-16. https://doi.org/10.7589/0090-3558-17.1.153

Woodman, N., Timm, R.M., Slade, N.A., Doonan, T.J., 1996. Comparison of traps and baits for censusing small mammals in Neotropical lowlands. Journal of Mammalogy. 77,274-281. https://doi.org/10.2307/1382728

World Wildlife Fundation., Fundación Omacha. \& Center for Environmental Science University of Maryland. 2016. Cuenca del río Orinoco 2016 - Reporte de salud. Instituto de Investigación de los Recursos Naturales Alexander Von Humboldt, Bogotá, Colombia 26

Zar, J.H., 2010. Biostatistical analysis. Pearson Prentice Hall, New Jersey, E.E.U.U. 944

Mammalogy Notes | Notas Mastozoológicas

Sociedad Colombiana de Mastozoología

Vol. 5 Núm. 2| 2019 


\section{Artículo | Article}

MAMMALOGY NOTES I NOTAS MASTOZOOLÓGICAS

ANEXOS

Anexo 1. Especímenes recolectados en el campus de la Universidad de los Llanos y depositados en el Museo de Historia Natural Unillanos (MHNU).

\begin{tabular}{llcc}
\hline Numero de catalogo & Especie & Fecha de captura & Coordenadas \\
\hline MHNU-M-291 & Mus musculus & $2016-10-05$ & $4.0725,-73.5827$ \\
MHNU-M-292 & Rodentia & $2016-06-05$ & $4.0725,-73.5827$ \\
MHNU-M-293 & Rattus rattus & $2016-10-10$ & $4.0725,-73.5827$ \\
MHNU-M-294 & Coendou prehensilis & $2015-02-02$ & $4.075,-73.581$ \\
MHNU-M-295 & Caluromys lanatus & $2016-10-16$ & $4.072,-73.586$ \\
\hline
\end{tabular}

Anexo 2. Mamíferos no voladores de la Universidad de los Llanos. Método de registro: cámara trampa (C), trampa Sherman (S), trampa Tomahawk (T), recorrido (R), individuos encontrados muertos (M), entrevista (E), museo (Mu). Estado de conservación (EC) según UICN (2016) y Minambiente (2017): Preocupación menor (PM), Vulnerable (VU).

\begin{tabular}{|c|c|c|c|c|c|c|c|c|}
\hline Taxón & $\mathbf{C}$ & $\mathbf{S}$ & $\mathbf{T}$ & $\mathbf{R}$ & $\mathbf{M}$ & $\mathbf{E}$ & Mu & EC \\
\hline \multicolumn{9}{|l|}{ DIDELPHIMORPHIA } \\
\hline Caluromys lanatus (Olfers 1818) & & & & & $\mathrm{X}$ & & & $\mathrm{PM}$ \\
\hline Didelphis marsupialis Linnaeus 1758 & $\mathrm{X}$ & $\mathrm{X}$ & $\mathrm{X}$ & $\mathrm{X}$ & $\mathrm{X}$ & $\mathrm{X}$ & & $\mathrm{PM}$ \\
\hline Philander opossum (Linnaeus 1758) & $\mathrm{X}$ & & & & & & $\mathrm{X}$ & $\mathrm{PM}$ \\
\hline \multicolumn{9}{|l|}{ CINGULATA } \\
\hline Dasypus novemcinctus (Linnaeus 1758) & $\mathrm{X}$ & & & & & $\mathrm{X}$ & & $\mathrm{PM}$ \\
\hline \multicolumn{9}{|l|}{ PILOSA } \\
\hline Choloepus didactylus (Linnaeus 1758) & & & & & & $\mathrm{X}$ & & $\mathrm{PM}$ \\
\hline Tamandua tetradactyla (Linnaeus 1758) & $\mathrm{X}$ & & & & & & & $\mathrm{PM}$ \\
\hline Myrmecophaga tridactyla Linnaeus 1758 & $\mathrm{X}$ & & & $\mathrm{X}$ & & $\mathrm{X}$ & & VU \\
\hline \multicolumn{9}{|l|}{ CARNIVORA } \\
\hline Cerdocyon thous (Linnaeus 1766) & $\mathrm{X}$ & & & & & & & $\mathrm{PM}$ \\
\hline Procyon cancrivorus (Cuvier 1798) & & & & & & $\mathrm{X}$ & & $\mathrm{PM}$ \\
\hline \multicolumn{9}{|l|}{ CETARTIODACTYLA } \\
\hline Odocoileus virginianus (Boddaert 1784) & $\mathrm{X}$ & & $\mathrm{X}$ & & & $\mathrm{X}$ & & $\mathrm{PM}$ \\
\hline \multicolumn{9}{|l|}{ PRIMATES } \\
\hline Aotus brumbacki (Hershkovitz 1983) & & & & & & $\mathrm{X}$ & & VU \\
\hline Saimiri cassiquiarensis (Linnaeus 1758) & & & & $\mathrm{X}$ & & & & $\mathrm{PM}$ \\
\hline Sapajus apella (Linnaeus 1758) & & & & $\mathrm{X}$ & & & & $\mathrm{PM}$ \\
\hline Plecturocebus ornatus (Gray 1866) & & & & $\mathrm{X}$ & & $\mathrm{X}$ & & VU \\
\hline \multicolumn{9}{|l|}{ RODENTIA } \\
\hline Notosciurus cf. granatensis (Wagner 1842) & $\mathrm{X}$ & & & $\mathrm{X}$ & & & & $\mathrm{PM}$ \\
\hline Coendou prehensilis (Linnaeus 1758) & & & & & $\mathrm{X}$ & $\mathrm{X}$ & & PM \\
\hline Cuniculus paca (Linnaeus 1766) & $\mathrm{X}$ & & & & & $\mathrm{X}$ & & $\mathrm{PM}$ \\
\hline Dactylomys dactylinus (Desmarest 1817) & & & & $\mathrm{X}$ & & $\mathrm{X}$ & & $\mathrm{PM}$ \\
\hline
\end{tabular}

Mammalogy Notes | Notas Mastozoológicas

Sociedad Colombiana de Mastozoología

Vol. 5 Núm. 2| 2019 


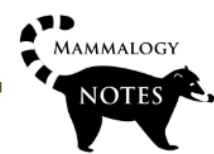

MAMMALOGY NOTES I NOTAS MASTOZOOLÓGICAS

Anexo 3. Fotografías de mamíferos en la Universidad de los Llanos registradas por estudiantes y docentes. Didelphis marsupialis (a), Philander opossum (b), Coendou prehensilis (c), Aotus brumbacki (d), Choloepus didactylus (e), Dactylomys dactylinus (f). Mamíferos capturados en cámaras trampa: Dasypus novemcinctus (g), Myrmecophaga tridactyla (h), Tamandua tetradactyla (i), Cerdocyon thous (j), Notosciurus cf. granatensis (k), Cuniculus paca (1), Procyon cancrivorus (m), Odocoileus virginianus (n).

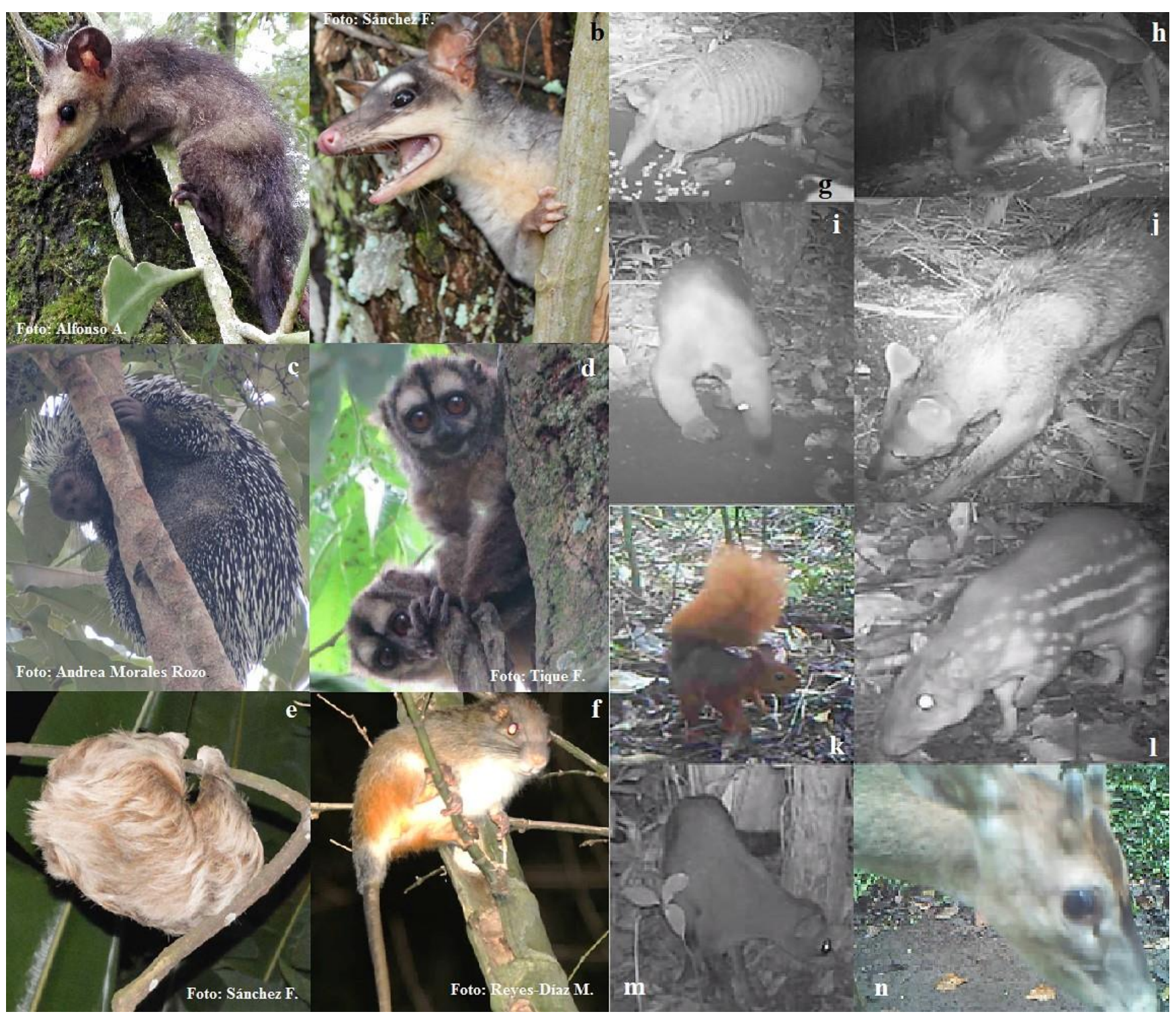

